\title{
eTutor: Um Ambiente de Aprendizagem Interativo
}

\author{
Rafael S. Santos ${ }^{1}$, Bruno N. Luz ${ }^{1,2}$, Valéria F. Martins ${ }^{3}$, Marcelo P. Guimarães ${ }^{1,4}$ \\ ${ }^{1}$ Programa de Mestrado em Ciência da Computação - Faculdade Campo Limpo Paulista \\ (FACCAMP) - Campo Limpo Paulista - SP - Brasil \\ ${ }^{2}$ Campus Boituva - Instituto Federal de São Paulo (IFSP) - Boituva - SP - Brasil \\ ${ }^{3}$ Faculdade de Computação e Informática - Universidade Presbiteriana Mackenzie \\ ${ }^{4}$ Universidade Federal de São Paulo (UNIFESP) - São Paulo - Brasil \\ rafael@renovaci.com, nogueira_luz@ifsp.edu.br, \\ valfarinazzo@hotmail.com, marcelodepaiva@gmail.com
}

\begin{abstract}
The teaching-learning process is influenced by several issues, such as the quantity of students per class and the complexity level of the course. a strategy for integrated monitoring of individual students to a computational tool (eTutor) which allows the teacher carry out appropriate actions in order to improve the teaching-learning process. ETutor monitors the current learning state and sends notification to the teacher, enabling him/her to offer assistance. This paper also presents the results reached by a course based on eTutor. This environment is based on Interactive Learning Objects.
\end{abstract}

Resumo. O processo de ensino-aprendizagem é influenciado por diversas variáveis, tais como a quantidade de alunos por classes e o nível de complexidade dos conceitos desenvolvidos. Este artigo apresenta uma estratégia de acompanhamento individual de alunos integrada a uma ferramenta computacional (eTutor), que possibilita ao professor realizar ações condizentes, aproximando-o do aluno e potencializando o desenvolvimento de processos de ensino-aprendizagem. $O$ eTutor monitora o estado atual de aprendizagem e notifica o professor para que ações apropriadas sejam tomadas. É apresentado também o resultado do uso do eTutor em um curso de extensão. A base desse ambiente são Objetos de Aprendizagem Interativos.

\section{Introdução}

Aprender é uma atividade fundamental e desafiadora para os seres humanos. O contexto institucional exige dedicação dos professores e dos alunos, mas proporciona muita satisfação, principalmente quando os alunos se apropriam do conhecimento e os aplicam em contextos diferentes dos abordados durante o processo de aprendizagem, envolvendose diretamente com as informações recebidas [Svinick e Mckeachie 2012].

Alguns alunos têm maior dificuldade do que outros durante a aprendizagem [Freire 1996, Zabala 1998], então a persistência de todos os envolvidos na criação de situações que apoiem o domínio de uma prática ou a compreensão de um assunto são fatores primordiais para que os objetivos sejam alcançados [Wells 1999]. Esta visão está apoiada no conceito de zona de desenvolvimento próximo de Vygotisky, exemplificado na frase: "a distância entre o nível de desenvolvimento atual determinado pela resolução 
independente de problemas e o nível de desenvolvimento potencial determinado pela resolução de problemas sob orientação ou em colaboração com parceiros mais capazes" [Vygotsky 1987, Vygotsky 1998].

Geralmente, os alunos que participam ativamente das aulas (expondo dúvidas, buscando resolver os exercícios propostos, não se ausentando e não desistindo) atingem o nível desejado de conhecimento em algum momento. Este é um dos pressupostos enfatizados por autores que discutem a zona de desenvolvimento próximo do aluno, com destaque à propriedade do aluno que permite uma aprendizagem mais fácil e mais apropriada [LeFrancois 2001, Fabes e Marin 2001]. Integrado a este aspecto, destaca-se o pressuposto da assistência, que enfatiza a importância da presença e assistência do professor, independentemente da proximidade física (ensino presencial ou a distância). Devido à disparidade de tempo de aprendizagem dos alunos, é muito importante que o professor esteja atento a cada aluno, esclareça todas as dúvidas e busque adequações no tratamento do conteúdo para aproximá-lo das possibilidades e potencialidades de aprendizagem do aluno. Existem várias atitudes que os professores podem realizar a fim de potencializar a aprendizagem, por exemplo, ele pode coletar individualmente as dúvidas e compartilhar com todos, pois a maioria dos questionamentos é comum a todos; e analisar a compreensão dos alunos sobre os assuntos durante a explicação, pois isto pode motivar a necessidade da mudança na forma de ensino, a fim de alcançar uma maneira que o aluno entenda o problema e a solução [Vygotsky 1978].

Acompanhar os alunos individualmente é um desafio para os professores, pois tal ação é influenciada por questões como, por exemplo, a quantidade de alunos da turma e as diferenças no tempo de aprendizagem de cada aluno [Freire 1996, Zabala 1998]. Portanto, as ferramentas tecnológicas são instrumentos que podem auxiliar no acompanhamento e nas orientações dos alunos, oferecendo soluções como: facilitar o acompanhamento das atividades em execução por cada aluno de maneira síncrona e/ou assíncrona; facilitar a difusão de esclarecimento de dúvidas coletivas e individuais; integrar espaços coletivos e individuais de produção; e oferecer auxílios a cada aluno conforme o estágio de aprendizagem.

Este artigo apresenta o eTutor, uma ferramenta computacional que auxilia o acompanhamento individual dos alunos através de ações interativas, favorecendo o desenvolvimento de situações de aprendizagem que possam viabilizar a assistência educacional ao aluno. Esta assistência decorre da observação das ações realizadas pelo estudante que predizem a necessidade de intervenção no processo de aprendizagem de forma apropriada. Esta intervenção pode acontecer, por exemplo, através da sugestão de leitura de um texto ou material de apoio; ou da demonstração, de perguntas-guia ou da introdução de elementos da solução da tarefa [Vygotsky 1987]. Assim, esse ambiente atua diretamente sobre as atividades do aluno, monitorando o estado atual e gerando ações automáticas; e sobre a condução da aula pelo professor, alertando-o e facilitando o compartilhamento de conteúdo. A motivação deste trabalho se deu através da percepção dos autores (professores) que melhores resultados são alcançados com alunos sempre que apoio individualizado é ofertado, mesmo em salas com uma quantidade grande de alunos [Bloom 1984].

A base educacional e tecnológica deste projeto é a criação dos Objetos de Aprendizagem Interativos (OAI) - por exemplo, exercício e explicações - capazes de monitorar as atividades realizadas pelos alunos, oferecendo ajuda e alertando o professor 
de acordo com as dificuldades específicas do aluno. Esses OAI são uma extensão dos Objetos de Aprendizagem tradicionais, no caso do padrão SCORM. Com a utilização do ambiente proposto, os OAI são criados, gerenciados, distribuídos e reutilizados. O professor cria os OAI e os distribui entre os alunos. Desta forma, o ambiente de aprendizagem monitora as ações realizadas nos objetos individuais, o que permite relatar ao professor, o estado atual e, além disso, fornece auxílio individual a cada aluno conforme o estado, como, por exemplo, sugere um vídeo explicativo.

Esse ambiente, que está apoiado na estratégia educacional de acompanhamento e com suporte na teoria de zona de desenvolvimento próximo, tem a finalidade de potencializar as ações de ensino do professor, aproximando-o do aluno. Não faz parte deste trabalho adaptar o conteúdo da disciplina, realizando ações como, por exemplo, propor um exercício de reforço para o aluno de maneira automática. Como o professor acompanha o estado atual de aprendizagem de cada aluno, cabe a ele este tipo de ação na configuração dos objetos. Contudo, o eTutor utiliza configurações prévias de cada OAI e compara com o estado atual do objeto para oferecer ajuda aos alunos e notificar o professor (módulo de tomada de decisão).

O artigo está organizado da seguinte forma: a seção 2 traz as definições dos OAI desenvolvidos e utilizados; a seção 3 expõe a criação do ambiente de aprendizagem, o eTutor; a seção 4 traz explicações sobre o módulo de tomada de decisão, que avalia o estado dos objetos e gera ações no ambiente; a seção 5 apresenta a metodologia de aplicação dos testes e validação feitos em um curso de extensão; a seção 6 traz análises e discute o comportamento do sistema de monitoramento; e, por fim, a seção 7 apresenta as conclusões.

\section{Objetos de Aprendizagem Interativos}

O avanço da internet tem impulsionado vários mecanismos capazes de proporcionar o aprendizado e conhecimento através de conjuntos de materiais didático-pedagógicos, transformando-os em unidades de aprendizagem passíveis de reutilização e compartilhamento, denominados Objetos de Aprendizagem (OAs) [Downes 2001].

Segundo L'Allier (1997), um OA é definido como "a menor estrutura instrucional independente que contém um Objetivo, uma Atividade de Aprendizagem", consistido em um componente digital, base de um curso, unidade ou lição que pode ser reutilizado para criar outras estruturas instrucionais únicas. Tendo suas principais características descritas como: 1) Flexibilidade - desenvolvido para ser reutilizado; 2) Personalização personalização de conteúdos permitindo a sua recombinação; 3) Interoperabilidade permite a definição de especificações de design, de desenvolvimento e de apresentação baseadas em necessidades da organização; e 4) Aumento de significância do conhecimento - com a reutilização de um OA várias vezes e em diferentes situações, seu conteúdo é consolidado naturalmente com o passar do tempo.

Na proposta desenvolvida neste trabalho, na utilização do eTutor, um OA deve ser considerado se apresentar uma sequência de fases que garantam a interação, além, obviamente, dos demais pontos e características destacados anteriormente. Para tanto, foi necessário expandir o padrão SCORM, adicionando elementos (como, por exemplo, tempo sem interação, nível de dificuldade, ajuda através de texto, vídeo ou áudio) que permitam o acompanhamento do estado atual do objeto. Assim, Objetos de Aprendizagem Interativos foram criados. Com o olhar voltado para a interação e 
principalmente para os benefícios agregados que ela proporciona no processo de ensino e aprendizagem, Kemezinski et.al (2012) já descreviam uma metodologia para construção de objetos de aprendizagem interativos, porém, se limitavam a este processo sem a preocupação de se estruturar a construção do objeto baseada em padrões, como o SCORM. Para os autores deste trabalho, para ser considerado um OAI, tal objeto deve permitir a interação conforme ilustra a Figura 1. Nela, o OAI é pré-configurado pelo professor com parâmetros como, por exemplo, o período de tempo para que o professor será alertado que o aluno não está interagindo com o objeto; e quando dicas, como textos e vídeos, serão oferecidas para o aluno. Ao monitorar o estado do objeto, o eTutor pode realizar diversas ações, como oferecer ajuda e/ou alerta ao professor, que poderá visualizar o estado atual do objeto do aluno e, se desejar, iniciar uma interação via chat.

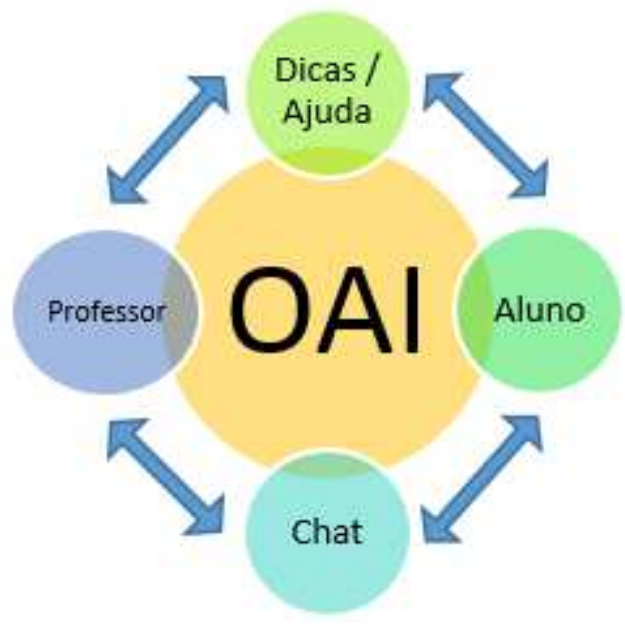

Figura 1. Operação de um Objeto de Aprendizagem Interativo

O eTutor foi desenvolvido de forma a garantir a personalização e interação dos OAIs, sendo desenvolvido seguindo a definição destes objetos, garantindo, por exemplo, a utilização de chats, dicas e controle do objeto em tempo real. Como os OAIs são uma extensão do SCORM, então podem ser exportados para ferramentas compatíveis; contudo perdem as funcionalidades de interação.

\section{3. eTutor - Ambiente de Aprendizagem Interativo}

A Figura 2 apresenta a interface do eTutor com um OAI carregado. $\mathrm{O}$ foco deste ambiente é o oferecimento de soluções que permitam o acompanhamento individual dos alunos, a fim de estimular situações de aprendizagem capazes de proporcionar uma melhor assistência educacional, independente da distância física, através de iniciativas que promovam a interação entre os envolvidos. Cada OAI é composto por atributos, como nome, link e objetos multimídia (áudio, vídeo e texto); ações, como os alertas enviados para o professor quando não ocorre interação com a OAI; e os tipos de ajudas a serem oferecidas aos alunos. 


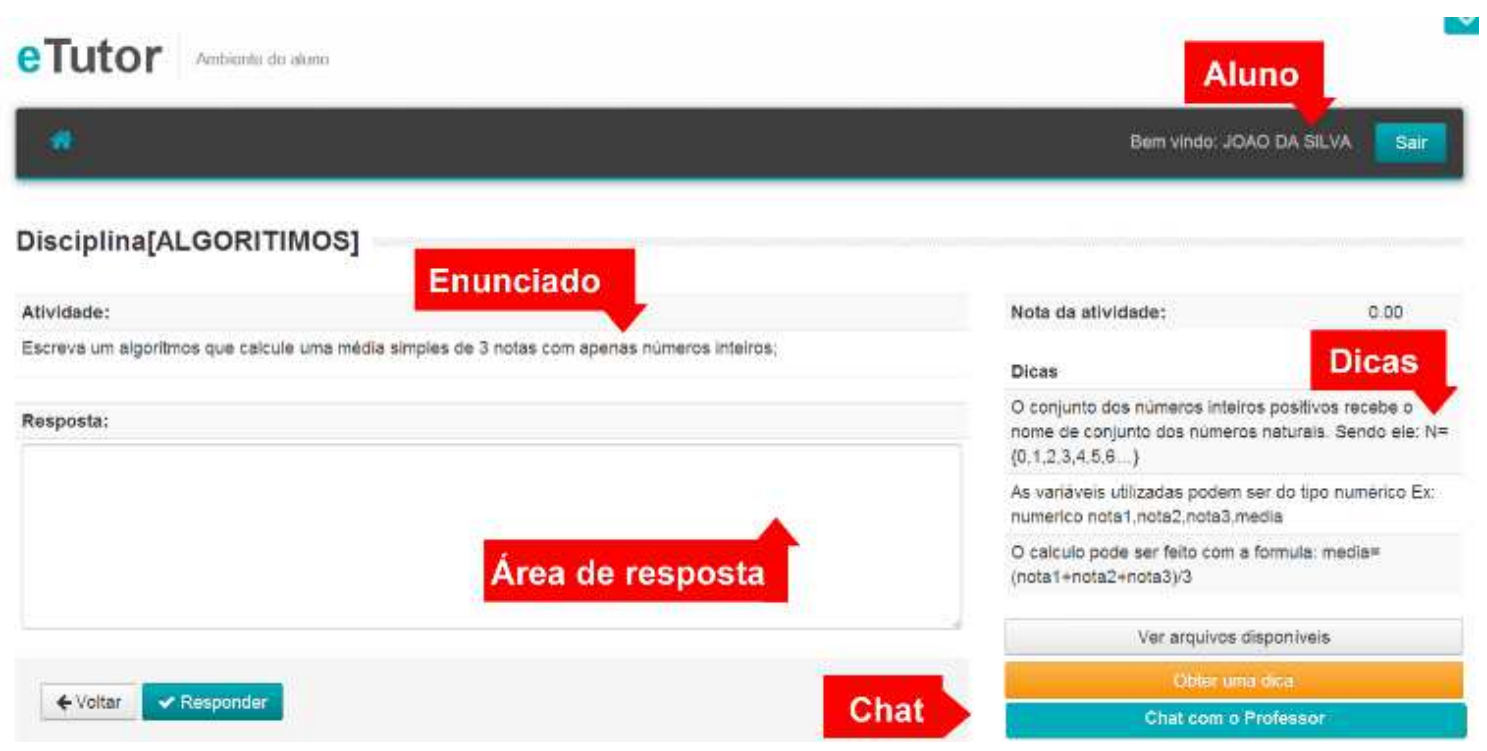

Figura 2. eTutor - Interface Principal - Aluno

$\mathrm{Na}$ ferramenta, o professor cria os OAIs e os distribui entre os alunos. Então, o ambiente monitora as ações realizadas sobre os objetos. A seguir, são apresentados alguns exemplos de situações que geram ações no ambiente:

- Envio de alerta ao professor caso o aluno fique por um determinado período de tempo sem interagir com o OAI.

- Disponibilização de algum tipo de ajuda ao aluno (por exemplo, um vídeo ou texto explicativo).

- Geração de um alerta ao professor caso o aluno esteja solicitando ajuda e já acessou todos os outros tipos de ajuda (texto, vídeo, áudio).

Ao professor é disponibilizada uma interface específica (Figura 3), que permite o monitoramento de todos os alunos. Nela, o professor pode solicitar uma cópia do OAI de um aluno, o que permitirá sua verificação sobre o estado atual da resolução do exercício. Se o professor tiver a percepção da necessidade de uma intervenção, ele poderá se comunicar com o aluno (diretamente, no caso de ensino presencial; ou via chat/voz, no caso de ensino a distância).

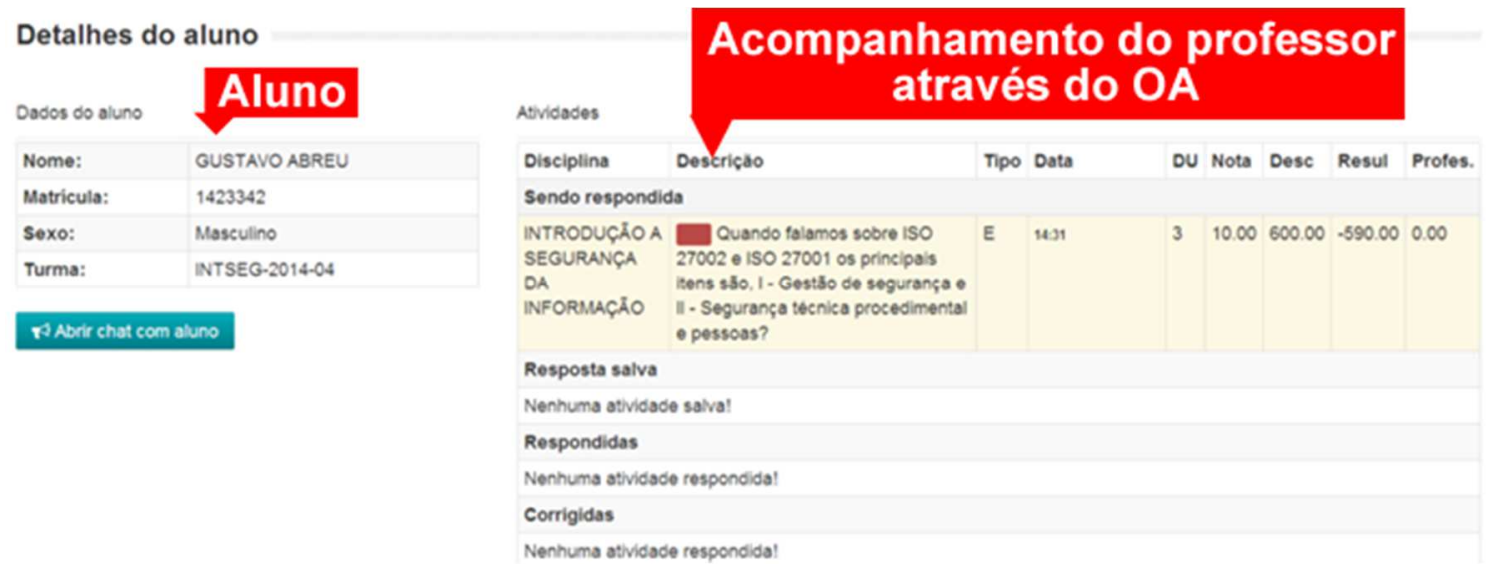

Figura 3. Análise do OAl de um aluno apresentado para o professor.

Um dos desafios deste ambiente é receber como entrada o estado do OAI e acionar a ação apropriada. Os parâmetros de comportamento de cada objeto são configurados pelo 
próprio professor no momento da criação do OAI, como, por exemplo, "se o aluno ficar mais de 3 minutos sem interagir com o objeto, o sistema deve sugerir uma determinada dica”. Estas entradas são utilizadas pelo módulo de tomada de decisão a fimm de que ações pré-configuradas ocorram, como o oferecimento de uma dica ou o alerta ao professor.

\subsection{Módulo de Tomada de Decisão}

O módulo de tomada de decisão foi implementado utilizando lógica Fuzzy como descreve Guimarães, Martins e Dias (2013). Nele são configurados os parâmetros que servem como base para a instanciação de ações automáticas, ou seja, as ações que serão realizadas conforme o estado atual do objeto; por exemplo, o professor pode configurar o eTutor para oferecer dicas, tal como um vídeo, se o aluno não adicionar nenhuma informação em 5 minutos em um exercício descritivo; e se a situação persistir, o professor deve ser alertado.

Este módulo se assemelha ao modelo adaptativo de Seters, Ossevoort, Tramper e Goedhart (2012), que integra informações anteriores (como tempo de inatividade e dicas utilizadas) com a finalidade de selecionar o conteúdo de aprendizado apropriado para apresentar ao aluno, ou ainda, efetivar ações como comunicar o professor, baseado em variáveis de entrada e de saída, que representam o resultado final da avaliação de desempenho do aluno (e a intervenção ou não no processo de aprendizado).

Para a implementação do módulo de tomada de decisão no eTutor, as seguintes variáveis de entrada do OAI são consideradas para o disparo da ação apropriada:

- Tempo sem interagir: significa quanto tempo o aluno ficou sem interagir com o OAI, seja ele o exercício propriamente dito ou instrumentos complementares de aprendizagem, tais como um vídeo ou um texto.

- Quantidade de caracteres inseridos: porcentagem de caracteres que o usuário digitou em função de um número médio de caracteres por exercício, definido pelo professor. Esta quantidade pode ser baixa, média ou alta.

- Pedidos de ajuda: significa a quantidade de pedidos já realizada pelo aluno, que pode ser baixo, médio ou alto.

- Dificuldade do exercício proposto: nível do exercício que o professor propõe, que pode ser rotulado como fácil, intermediário ou difícil.

Considerando essas variáveis de entrada, o eTutor determina o nível de ajuda a ser oferecido ao aluno: Nível 0 - nenhuma ajuda; Nível 1 - áudio, vídeo apresentado ao aluno como material de apoio sobre o tema; Nível 2 - rever exercício - rever um exercício comentado sobre um tema que tenha sido apresentado pelo professor; Nível 3 - ajuda monitor, ou seja, o monitor da disciplina é designado para ir ao computador aonde está o aluno; Nível 4 - ajuda professor, ou seja, é requisitada a ajuda presencial do professor.

Faz parte também desse módulo fatores de penalidades associados aos níveis de dicas utilizados. Estas penalidades são também configuradas, opcionalmente, pelo professor ao criar o OAI, que descontará um valor da nota associada à atividade. 


\section{Metodologia de Aplicação dos Testes}

O eTutor foi desenvolvido utilizando os OAI e o módulo de tomada de decisões de forma a garantir a interatividade proposta no trabalho. O ambiente foi validado em um curso de "Introdução a Segurança da Informação" (curso de extensão - 16 horas). A turma possuía cinco alunos matriculados, o que permitiu e garantiu um ambiente controlado, atendendo assim a validação inicial do sistema.

Para a preparação dos testes, o professor e os alunos foram cadastrados no eTutor pelo administrador. O professor recebeu orientações dos autores acerca da ferramenta, navegação e construção dos objetos no sistema.

No curso avaliado, o professor criou cinco OAIs nos moldes de um questionário com múltipla escolha e categorizados em níveis de dificuldade. Cada objeto contou com, no mínimo, três dicas, que estavam à disposição dos alunos para consulta, e, também, préconfiguradas para disponibilização automática conforme dados de entrada do professor.

Ao final do curso, o grupo foi submetido a um questionário, com intuito de mapear a utilização, navegabilidade e interação proporcionada pelo ambiente. Estes resultados são descritos na próxima seção. O questionário aplicado aos alunos foi composto de questões que envolvem os aspectos como a facilidade de uso do ambiente, interação e acompanhamento do professor com a disponibilização de dicas e auxílios.

\section{Análise e Discussão}

Como resultado dos testes de validação, os usuários que participaram foram: cinco alunos e um professor. As questões aplicadas aos alunos foram tabuladas e as respostas apresentadas nas tabelas 1 e 2, conforme os fatores mapeados: facilidade de uso; e disponibilização de dicas. Para as questões com avaliações qualitativas, foi atribuída uma escala de 1 a 5, sendo: nota 1 (pouca influência), 2 (regular), 3 (bom), 4 (ótimo) e 5 (excelente).

Tabela 1. Respostas do questionário dos alunos referentes as dicas.

\begin{tabular}{l|c|c|c|c|c}
\multicolumn{1}{c|}{ DICAS } & Aluno 1 & Aluno 2 & Aluno 3 & Aluno 4 & Aluno 5 \\
\hline $\begin{array}{l}\text { Durante a utilização do objeto de aprendizagem, } \\
\text { você recebeu dicas na atividade? }\end{array}$ & Sim & Sim & Sim & Sim & Sim \\
\hline $\begin{array}{l}\text { Durante a utilização do objeto de aprendizagem, } \\
\text { você interagiu com o professor solicitando ajuda? }\end{array}$ & Não & Não & Sim & Sim & Sim \\
\hline $\begin{array}{l}\text { Em relação a possibilidade de solicitar e receber } \\
\text { dicas durante a atividade. }\end{array}$ & 4 & 5 & 4 & 4 & 4 \\
\hline $\begin{array}{l}\text { Em relação aos arquivos disponibilizados junto } \\
\text { com as dicas com intuito de auxiliar na atividade. }\end{array}$ & 4 & 1 & 5 & 4 & 4 \\
\hline $\begin{array}{l}\text { Em relação a possibilidade de interação no Chat } \\
\text { com o professor durante a atividade. }\end{array}$ & 3 & 3 & 4 & 4 & 4
\end{tabular}

Em todos os casos os alunos receberam dicas automáticas do ambiente conforme pré-configurado pelo professor. Destes, apenas um considerou o material de apoio extra com pouca influência no resultado, os demais consideraram como ótimo e excelente a utilização do recurso proporcionado pela interação do OAI.

A interação com o professor na forma de solicitação de ajuda ocorreu em $60 \%$ dos casos, e os alunos que a fizeram julgaram como ótima a possibilidade de solicitar e receber dicas durante a atividade. Da mesma forma, estes alunos (60\%) consideraram como ótima a interação via recurso de chat com o professor. 
Tabela 2. Respostas do questionário dos alunos referentes a facilidade de uso.

\begin{tabular}{l|c|c|c|c|c}
\multicolumn{1}{c|}{ FACILIDADE DE USO } & $\begin{array}{c}\text { Aluno } \\
\mathbf{1}\end{array}$ & $\begin{array}{c}\text { Aluno } \\
\mathbf{2}\end{array}$ & $\begin{array}{c}\text { Aluno } \\
\mathbf{3}\end{array}$ & $\begin{array}{c}\text { Aluno } \\
\mathbf{4}\end{array}$ & $\begin{array}{c}\text { Aluno } \\
\mathbf{5}\end{array}$ \\
\hline $\begin{array}{l}\text { Em relação a facilidade de uso do objeto de aprendizagem } \\
\text { (não teve dificuldade em responder/interagir com o objeto). }\end{array}$ & 4 & 5 & 4 & 4 & 4 \\
\hline $\begin{array}{l}\text { Você já tinha realizado alguma atividade de questionário } \\
\text { através de Objetos de Aprendizagem? }\end{array}$ & Sim & Sim & Sim & Sim & Sim
\end{tabular}

Mesmo os alunos que não solicitaram ajuda do professor, consideraram a possibilidade de fazê-lo como ótima e excelente, e indicaram o uso do chat como bom. Este perfil de aluno, que apresenta um desenvolvimento mais individualizado no aprendizado, se encaixa dentro das inteligências múltiplas descritas por Gardner (2002), com maior autonomia, o que embasa e justifica os fatores de independência demonstrados nos resultados obtidos, o que, de forma alguma, desmerece a opção disponibilizada pelo ambiente.

Nesses testes, o ambiente eTutor atendeu as expectativas em relação ao acompanhamento dos alunos pelo professor, garantindo um acompanhamento conforme as instâncias dos OAIs de cada aluno eram utilizadas, permitindo ao professor uma visão clara e imediata do andamento do aluno nas atividades. Quando a instância do OAI do aluno atingia algum dos parâmetros definidos pelo professor, o sistema oferecia dicas diretamente ao aluno, bem como alertas visuais ao professor que teria, à sua disposição, meios para interagir com o aluno, tais como: ofertar dicas de forma síncrona; mediação via chat; e disponibilização de arquivos de ajuda.

\section{Conclusão}

Os avanços tecnológicos, tais como os que permitem a transmissão de dados, impulsionam o uso de computadores no processo de ensino-aprendizagem, provendo soluções, como chat e web conferência, e servem como item de aproximação entre os envolvidos [Tori 2010]. Neste contexto, além de uma solução tecnológica adequada é necessária também uma estratégia educacional. É papel do professor acompanhar os alunos a fim de diagnosticar o contexto de ensino-aprendizagem; desta forma é possível tomar decisões, realizar ações e reavaliá-las constantemente encaminhando-as em uma maneira adequada [Zabala 1998].

Este trabalho apresentou uma proposta de solução de ambiente educacional que possibilita o acompanhamento individual dos alunos, permitindo que o professor crie os OAIs, definindo parâmetros que possibilitam um monitoramento pelo ambiente sob o seu estado atual, que é retransmitido para o professor. O acompanhamento do estado dos objetos permite que o professor realize ações direcionadas a cada aluno. Além disso, faz parte dos objetos ações pré-definidas, como a oferta de ajuda e dicas. É importante notar que a ferramenta busca prover um acompanhamento das atividades dos alunos e não escalabilidade (aumentar a quantidade de alunos atendidos por um professor). Após seu primeiro teste com uma turma, o ambiente é avaliado como positivo, uma vez que os alunos se sentiram atendidos e acolhidos pela atenção direta do professor, e este, mais próximo dos alunos e do desenvolvimento e percepção dos conteúdos pelos mesmos. 
Destaca-se a interação proporcionada na disponibilização das dicas e alertas ao professor sobre o estado do aluno, fatores que garantem o acompanhamento citado ao longo do trabalho, com vistas a um resultado melhor no processo de ensino e aprendizagem mediado pelas Tecnologias de Informação e Comunicação (TICs).

A interação dos objetos construídos são uma excelente opção para todas as áreas do conhecimento ajudando e aumentando a absorção e aprendizado. Estudos e pesquisas ainda podem ser realizados para avaliar qual técnica de adaptação é mais eficiente. Assim, poderá ser utilizado no futuro: (i) novos parâmetros de monitoramento e controle; (ii) utilização de Redes Neurais ou Sistemas de Inteligência Artificial, levando em conta o desempenho, confiabilidade e o grau de dificuldade de implementação de cada uma; (iii) testes envolvendo turmas com uma quantidade maior de alunos.

\section{Referências}

Arias, R. A., Panhan, A. M., Breda, G. D., Zarpelão, B. B., Mendes, L.S. (2012) "Avaliação Multidimensional Baseada em Lógica Difusa para Ambientes de Ensino Mediado por Computador". Revista Brasileira de Informática na Educação”, Vol. 20, Número 3, pp.74-84.

Barbosa, S. D., e Silva, B. S. (2010). “Interação Humano-Computador”. Rio de Janeiro: Campus-Elsevier.

Beck, K., Andres, C. (2004). "Extreme programming explained: embrace change". Addison-Wesley Professional.

Beck, K. (1999) "Embracing change with extreme programming". Computer, v. 32, n. 10, pp. 70-77.

Bloom, B. S.(1984) The 2 Sigma Problem: The search for methods of group instruction aseffective as one-to-one tutoring". In: Educational Researcher. [S.l.]: [s.n.], p. 4-16.

Botturi, L., Derntl, M., Boot, E., e Figl, K. (2006). "A Classification Framework for Educational Modeling Languages in Instructional Design”. Proceedings of The 6th IEEE International Conference on Advanced Learning Technologies, (p. 5). Kerkrade

Costa, K. C. F., Harb, M. P. A. A., Brito, S. R., Favero, E. L. (2006). “Acompanhamento do estudante em ambientes de aprendizagem utilizando Lógica Fuzzy". Anais do XXVI Congresso da SBC.WIE 1 XII Workshop de Informática na Escola. Campo Grande, MS.

Downes S. (2001). "Learning Objects: Resources for Distance Education Worldwide". In International Review of Research in Open and Distance Learning. Volume 2 (1).

Fabri, J. A. and Fabri, M. G. (2002). "Ferramenta Fuzzy para Acompanhamento do Desempenho dos alunos nos Cursos a Distância". In XXII Congresso da Sociedade Brasileira de Computação. Workshop de Informática na Escola. Campinas.

Freire, P. (1996) "Pedagogia da Autonomia: saberes necessários à prática educativa" São Paulo: Paz e Terra.

Gardner, H. (2002) "Inteligência: um conceito reformulado". São Paulo: Objetiva, Estruturas da mente: a teoria das inteligências múltiplas. Porto Alegre: Artmed. 
Guimarães, M. P., Martins, V. F., Dias, D. C. (2013) "Uso de Lógica Fuzzy no Auxílio ao Acompanhamento Automático de Alunos utilizando um Ambiente de Aprendizagem". In: XXIV Simpósio Brasileiro de Informática na Educação (SBIE 2013). DOI: 10.5753/CBIE.SBIE.2013.707.

Kemezinski, A, Costa, I. A., Wehrmeister, M. A., Hounsell, M. S. e Vahldick, A. (2012) "Metodologia para Construção de Objetos de Aprendizagem Interativos", Anais do $23^{\circ}$ Simpósio Brasileiro de Informática na Educação (SBIE 2012), ISSN 2316-6533. Rio de Janeiro, 26-30 de Novembro de 2012.

L'Allier, J. J. (1997) “A Frame of Reference: NETg's Map to Its Products". Their Structures and Core Beliefs.

LeFrancois, G. (2001) "Of children: An introduction to child and adolescent development", 9th ed. Belmont, CA: Wadsworth/Thomsen.

Kenski, V. M. (2003) "Tecnologias e ensino presencial e a distância”. Série Prática Pedagógica. Campinas: Papirus. ISBN 8530807081.

Malvezzi, W. R., Mourão, A. B., Bressan, G. (2010) "Uma Ferramenta Baseada em Teoria Fuzzy para o Acompanhamento de Alunos Aplicado ao Modelo de Educação Presencial Mediado por Tecnologia". Anais do SBIE 2010. XXI Simpósio Brasileiro de Informática na Educação, João Pessoa. 10 pages.

Rissoli, V. R. V., Giraffa, L. M. M., \& Martins, J. D. P. (2008). “Sistema tutor inteligente baseado na teoria da aprendizagem significativa com acompanhamento fuzzy". Informática na educação: teoria \& prática, 9 (2).

Seters, J. R. V., Ossevoort, M. A., Tramper, J., Goedhart, M. J. (2012) "The influence of student characteristics on the use of adaptive e-learning material". In: Computers \& Education 58. Elsevier: pp. 942-952.

Sugeno, M. e Yasukawa, T. "Uma Abordagem Fuzzy-Logic-Based para Modelagem Qualitativa", IEEE Transactions on sistema fuzzy, v.1, pp. 7-31.

Svinick, M. and Mckeachie, W.J. (2012) 'Dicas de Ensino: estratégias, pesquisa e teoria para professores universitários", São Paulo: Cengage Learning. p. 201.

Tanenbaum, A. (2011) "Redes de Computadores”. (5 ed.) Pearson/Prentice Hall.

Tori, R. (2010) "Educação sem distância: as tecnologias interativas na redução de distâncias em ensino e aprendizagem”. São Paulo, Brasil: Editora Sena São Paulo.

Vygotsky, L. S. (1987) “Thinking and speech (n. Minick, Trans.)”, In R. W. Rieber \& A. S. Carton (Eds.). The collected works of L. S. Vygostky: Vol. 1. Problems of general psycology (pp. 39-285). New York: Plenum Press.

Vygotsky, L. S. (1998) “The problem of age (M. Hall, Trans.)”, In R. W. Rieber (Ed.The collected works of L. S. Vygotsky: (Vol. 5 Child psycology) (pp. 187-205). New York: Plenum Press.

Wells, G. (1999) "Dialogic inquiry: Towards a sociocultural practice and theory of education". Cambridge: Cambridge University Press.

Zabala, A. (1998) “A Prática Educativa: como ensinar”. Porto Alegre: Artmed.

Zadeh, L.A. (1996) "Fuzzy logic, neural networks, and soft computing”. World Scientific Series In Advances In Fuzzy Systems, pp. 775 - 782. 